\title{
Periostin suppresses in vivo invasiveness via PDK1/Akt/mTOR signaling pathway in a mouse orthotopic model of bladder cancer
}

\author{
CHUL JANG KIM ${ }^{1}$, YUKIHIRO TAMBE ${ }^{2}$, KEN-ICHI MUKAISHO ${ }^{3}$, HIROYUKI SUGIHARA $^{3}$, \\ SUSUMU KAGEYAMA $^{4}$, AKIHIRO KAWAUCHI ${ }^{4}$ and HIROKAZU INOUE ${ }^{2}$ \\ ${ }^{1}$ Department of Urology, Kohka Public Hospital, Kohka, Shiga 528-6024; Divisions of ${ }^{2}$ Microbiology and Infectious Diseases \\ and ${ }^{3}$ Molecular and Diagnostic Pathology; ${ }^{4}$ Department of Urology, Shiga University of Medical Science, \\ Otsu, Shiga 520-2192, Japan
}

Received September 25, 2015; Accepted February 14, 2017

DOI: $10.3892 / \mathrm{ol} .2017 .6004$

\begin{abstract}
Periostin is an extracellular matrix protein involved in the regulation of intercellular adhesion. The present study investigated the in vivo tumor suppressor function of periostin in a mouse orthotopic model of bladder cancer. Retroviral vectors were used to transfect human bladder cancer UMUC-3 cell line with periostin. Bladders of nude mice that were transurethrally instilled with periostin-expressing UMUC-3 cells were revealed to weigh less compared with bladders instilled with vector control cells. In total, five (83.3\%) of six vector control UMUC-3 bladder tumors exhibited histological evidence of muscle invasion. However, none of the five periostin-expressing UMUC-3 bladder tumors revealed muscle invasion. Thick edematous lesions were present in the submucosa of periostin-expressing UMUC-3 bladder tumors. The expression of periostin also suppressed in vitro cell invasiveness of UMUC-3 cells without affecting cellular proliferation. The level of phosphorylation of phosphoinositide-dependent kinase-1 (PDK1), protein kinase B (Akt) and S6 ribosomal protein, a downstream protein of mammalian target of rapamycin (mTOR) was decreased in periostin-expressing UMUC-3 cells compared with vector control cells. Treatment with $100 \mathrm{ng} / \mathrm{ml}$ recombinant human periostin protein also suppressed cell invasiveness and phosphorylation of PDK1, Akt and S6 in UMUC-3 cells, consistent with results using periostin-expressing UMUC-3 cells. Treatment with PDK1, Akt and mTOR inhibitors significantly suppressed UMUC-3 cell invasiveness. These results demonstrate that periostin suppresses in vivo and in vitro invasiveness of bladder cancer via the PDK1/Akt/mTOR signaling pathway. Periostin may be useful as a potent chemotherapeutic agent by suppressing bladder cancer invasiveness.
\end{abstract}

Correspondence to: Dr Hirokazu Inoue, Division of Microbiology and Infectious Diseases, Shiga University of Medical Science, Otsu, Shiga 520-2192, Japan

E-mail: hirokazu@belle.shiga-med.ac.jp

Key words: periostin, invasion, mTOR, bladder cancer, mouse model

\section{Introduction}

Periostin is an extracellular matrix (ECM) protein involved in the regulation of intercellular adhesion via interactions with other ECM proteins including fibronectin, tenascin-C, collagen $\mathrm{V}$ and periostin itself $(1,2)$. Periostin is highly expressed in early osteoblastic cells in vitro and in the periosteum and periodontal ligaments in vivo and has been revealed to serve a role in bone and tooth formation and the maintenance of structural integrity of these tissues (3). In addition, it has been reported that the expression of periostin correlates with the severity of heart failure (4) and that mechanical stress induces the expression of periostin in fibroblasts of the heart (5) and periodontal ligaments (6). Periostin has been frequently reported to be overexpressed in various types of human cancer cell lines and tissues, including breast, colon, ovarian and gastric cancer, non-small cell lung carcinoma and neuroblastoma (7). The overexpression of periostin by cancer stroma and/or neoplastic epithelium is generally associated with malignant phenotypes (7). However, this association was not identified in bladder cancer (8). Previously, we revealed that the downregulation of periostin mRNA expression was associated with higher grade human bladder cancer and that ectopic expression of periostin using a retroviral vector suppressed the in vitro invasiveness of human bladder cancer cells. We also demonstrated that periostin suppresses cell invasiveness in human bladder cancer cell lines via the downregulation of E-cadherin by suppressing protein kinase B (Akt) phosphorylation and Twist (9).

The present study investigated the in vivo tumor suppressor function of periostin in a mouse orthotopic model of bladder cancer and revealed that periostin suppressed the in vivo invasiveness of bladder cancer via the phosphoinositide-dependent kinase-1 (PDK1)/Akt/mammalian target of rapamycin (mTOR) signaling pathway.

\section{Materials and methods}

Orthotopic implantation of bladder cancer cells. All procedures were performed in compliance with the Ethical Guidelines for Animal Experimentation and Care and Use of Laboratory Animals at Shiga University of Medical Science 
(Otsu, Japan). The protocol was approved by the Committee on the Ethics of Research Center for Animal Life Science at Shiga University of Medical Science. The animals were housed in a specific pathogen-free room with controlled temperature (20-22 $\left.{ }^{\circ} \mathrm{C}\right)$, humidity (50-60\%) and a pre-set light/dark cycle $(12: 12 \mathrm{~h})$. Mice were allowed ad libitum access to food (CE-2; CLEA Japan, Inc., Tokyo, Japan) and water. A mouse orthotopic model of bladder cancer was used as described previously $(10,11)$. A total of 16,8 -week-old BALB/c-nu-nu female nude mice (ca $20 \mathrm{~g}$; CLEA Japan, Inc.) were used for the present study. A 24-gauze catheter was transurethrally inserted into the bladder of nude mice. In total, $100 \mu 10.2 \%$ trypsin in $0.02 \%$ EDTA was infused and retained in the bladder for $30 \mathrm{~min}$. Subsequent to trypsinization, bladders were washed with PBS (-). Subsequently, $100 \mu 1$ suspensions of serum-free medium containing $5 \times 10^{6}$ cancer cells were instilled into bladders. The urethra was ligated with a 4-0 nylon suture to ensure the retention of cancer cells. After $3 \mathrm{~h}$, the suture was removed, and bladder was evacuated by spontaneous voiding. A total of four weeks subsequent to the instillation of bladder cancer cells, mice were sacrificed under anesthesia and weighed. Bladders were excised and weighed. Opened bladder walls were then longitudinally cut, divided into four equal parts and histopathologically evaluated. Resected tumors that developed on the back of the mice were fixed with $10 \%$ formalin in PBS for $4 \mathrm{~h}$ and embedded in paraffin. Serial $3-\mu \mathrm{m}$ sections were used for histological evaluation using hematoxylin-eosin staining. Deparaffinization and rehydration of the sections was performed using 4 incubation steps of xylene and a series of $100 \%$ ethanol, $95 \%$ ethanol and twice $70 \%$ ethanol. Each step was performed for $5 \mathrm{~min}$ at room temperature. The sections were deionized using water for $15 \mathrm{~min}$ at room temperature. Subsequently, the sections were stained with hematoxylin (Merck KGaA, Darmstadt, Germany) for $4 \mathrm{~min}$ and eosin (Eosin Y; Merck KGaA) for $4 \mathrm{~min}$ at room temperature. To visualize these sections, we used a microscope (Eclipse E400; Nikon Corporation, Tokyo, Japan). Photos were captured using a digital camera system (DS-2Mv-L2; Eclipse E400; Nikon Corporation).

Mitotic counts in tumors developed in mouse urinary bladders. Mitotic counts were performed using a standard laboratory microscope (magnification, x400; Nikon ECLIPSE E400; Nikon Corporation, Tokyo, Japan). The mitotic activity index, mitotic figures/3 high-power fields (hpfs), of mouse orthotopic bladder tumors was determined using the number of mitotic figures in three randomly-selected hpfs.

Cell lines. The human bladder cancer UMUC-3 (American Type Culture Collection, Manassas, VA, USA) cell line was previously used in an experiment as the mouse orthotopic model of bladder cancer (11). UMUC-3 and 293T (American Type Culture Collection) were cultured in RPMI-1640 medium and Dulbecco's modified Eagle's medium (both from Nacalai Tesque, Inc., Kyoto, Japan), respectively, supplemented with $10 \%$ fetal calf serum (Sigma-Aldrich; Merck $\mathrm{KGaA})$, penicillin $(100 \mathrm{U} / \mathrm{ml})$ and streptomycin $(100 \mu \mathrm{g} / \mathrm{ml})$ at $37^{\circ} \mathrm{C}$ in a humidified $5 \% \mathrm{CO}_{2}$ atmosphere. The $293 \mathrm{~T}$ cell line was used for the preparation of recombinant retroviruses.
Preparation of recombinant retrovirus and virus infection. The pCXbsr retrovirus vector carrying human periostin complementary DNA (cDNA) and pCXbsr control vector (12) were transfected into 293T cells with the helper plasmid, pCL-ampho (13), using Lipofectamine-Plus reagent (Invitrogen; Thermo Fisher Scientific, Inc., Waltham, MA, USA) according to the manufacturer's protocol. The amphotropic retroviruses in the culture medium were collected $48 \mathrm{~h}$ subsequent to transfection, filtered and stored at $-80^{\circ} \mathrm{C}$ until use. For viral infection, $2 \times 10^{5}$ cells were seeded onto $60-\mathrm{mm}$ dishes and cultured overnight at $37^{\circ} \mathrm{C}$. Following polybrene treatment $(2 \mu \mathrm{g} / \mathrm{ml})$ for $30 \mathrm{~min}$, retroviruses were added to cell cultures and incubated for $1 \mathrm{~h}$ at $37^{\circ} \mathrm{C}$. Blasticidin-resistant colonies were selected subsequent to a 7-day incubation in media containing $5 \mu \mathrm{g} / \mathrm{ml}$ blasticidin (Invitrogen; Thermo Fisher Scientific, Inc.) prior to pooling and being used in additional assays.

Reverse transcription-polymerase chain reaction (RT-PCR) analysis. For RT-PCR, total RNA was isolated from cultured cells using TRIzol reagent (Invitrogen; Thermo Fisher Scientific, Inc.). Isolated RNA was used for first-strand cDNA synthesis using Superscript ${ }^{\mathrm{TM}}$ II Reverse Transcriptase (Invitrogen; Thermo Fisher Scientific, Inc.) and an Oligo (dT) $)_{12-18}$ primer (Invitrogen; Thermo Fisher Scientific, Inc.) for $50 \mathrm{~min}$ at $42^{\circ} \mathrm{C}$. cDNA was amplified by Taq DNA polymerase (Takara, Otsu, Japan) with primers specific for periostin: HP16S forward, 5'-GTGGTAGCACCTTCAAAGAAATCC-3' and HP22A reverse, 5'-GCAACTTCCTCACGGGTGTGTC-3' (12). PCR was performed with 30 cycles consisting of denaturation at $94^{\circ} \mathrm{C}$ for $30 \mathrm{sec}$, annealing at $55^{\circ} \mathrm{C}$ for $30 \mathrm{sec}$ and extension at $72^{\circ} \mathrm{C}$ for $30 \mathrm{sec}$, followed by a final extension for $7 \mathrm{~min}$. GAPDH primers were GAPDH-5F, 5'-ACCACAGTCCAT GCCATCAC-3' and GAPDH-3R, 5'-TCCACCACCCTGTTG CTTGTA-3'. For GAPDH, 30 cycles of PCR were performed with an annealing temperature of $60^{\circ} \mathrm{C}$. PCR products were separated by electrophoresis on $1.5 \%$ agarose gels containing ethidium bromide prior to imaging using an ultraviolet transilluminator and printgraph (AE6915; Atto, Co., Tokyo, Japan).

Pharmacological treatments. IL-6-hydroxymethyl-chiroinositol 2(R)-2-O-methyl-3-O-octadecylcarbonate (Akt inhibitor; Calbiochem; EMD Millipore, Billerica, MA, USA), 7-hydroxystaurosporine (UNC-01, PDK1 inhibitor, Sigma-Aldrich; Merck KGaA) and rapamycin (mTOR inhibitor, LC Laboratories, Woburn, MA, USA) were prepared in dimethyl sulfoxide (DMSO) and stored at $-20^{\circ} \mathrm{C}$. Cells were seeded at $1 \times 10^{6}$ cells per $60-\mathrm{mm}$ dish and incubated at $37^{\circ} \mathrm{C}$ for $16 \mathrm{~h}$. Inhibitors ( $0.1 \%$ of culture medium) were then added to culture media (PDK, 0.1 and $1 \mu \mathrm{M}$; Akt, 10 and $25 \mu \mathrm{M}$; mTOR, 5 and $20 \mathrm{nM}$ ), and cells were incubated at $37^{\circ} \mathrm{C}$ for $6 \mathrm{~h}$. In all experiments using inhibitors, the same volume of DMSO was added to control samples. Recombinant human periostin protein (r-periostin, SinoBiological, Inc., Beijing, China) was prepared in PBS(-) and stored at $-20^{\circ} \mathrm{C}$. In experiments using r-periostin, the same volume of PBS(-) was added to control samples.

In vitro invasion assay. The in vitro invasive potential of cells was determined using Matrigel $^{\mathrm{TM}}$ basement membrane matrix 
invasion chambers (chamber size, $6.4 \mathrm{~mm}$; membrane surface area, $0.3 \mathrm{~cm}^{2}$; pore size, $8 \mu \mathrm{m}$; BD Biosciences, Bedford, MA, USA) following the manufacturer's protocol. In total, $500 \mu \mathrm{l}$ cell suspension $\left(3 \times 10^{4}\right.$ cells $\left./ \mathrm{ml}\right)$ was added to each chamber. Chambers containing cells were incubated at $37^{\circ} \mathrm{C}$ for 2 days in a humidified $5 \% \mathrm{CO}_{2}$ atmosphere. Noninvasive cells were removed from membrane upper surfaces using cotton swabs. Invasive cells on membrane undersides were stained with Diff-Quik ${ }^{\mathrm{TM}}$ stain (Kokusai-Shiyaku, Kobe, Japan) and counted under a light microscope (TMS; Nikon Corporation). Each sample was assayed in triplicate in three independent experiments.

Tumorigenicity in nude mice with subcutaneous injection. The tumorigenicity of cancer cells was determined by injecting $3 \times 10^{6}$ cells (in $0.2 \mathrm{ml}$ ) subcutaneously into 6-week-old $\mathrm{BALB} / \mathrm{c}-\mathrm{nu} / \mathrm{nu}$ female mice. Tumorigenic potential was evaluated 3 weeks subsequent to inoculation. Mice were sacrificed under anesthesia, and tumors were excised and weighed.

Antibodies. Anti- $\alpha$-tubulin (clone, DM1A; \#T9026) and anti-Flag (clone, M2; \#F3165) monoclonal antibodies were purchased from Sigma-Aldrich; Merck KGaA. Anti-phospho Akt (T308 and S473, \#13038; D25E6 and D9E, \#4060) rabbit monoclonal, anti-Akt (\#9272) rabbit polyclonal, anti-phospho phosphatidylinositol 3 kinase [PI3K; p85 (Y458)/p55 (Y199)] rabbit polyclonal, anti-PI3K (p85; clone, 19H8; \#4257) rabbit monoclonal, anti-phospho phosphoinositide-dependent protein kinase 1 (PDK1; S241; \#3061) rabbit monoclonal, anti-PDK1 (\#3062) rabbit polyclonal, anti-phospho S6 ribosomal protein (S240/244, clone, D68F8; \#5364) rabbit monoclonal, anti-S6 ribosomal protein (clone, 5G10; \#2217) rabbit monoclonal and anti-Snail (clone, C15D3; \#3879) rabbit monoclonal antibodies were purchased from Cell Signaling Technology, Inc. (Danvers, MA, USA). The anti-E-cadherin (\#13116) mouse monoclonal antibody was purchased from BD Biosciences. The anti-Twist (clone H81; \#sc-15393) rabbit polyclonal antibody was purchased from Santa Cruz Biotechnology, Inc., (Dallas, TX, USA). All antibodies were uses at a dilution of 1:1,000.

Immunoblotting. Cells were lysed in Laemmli-SDS buffer containing $62.5 \mathrm{mM}$ Tris- $\mathrm{HCl}$ (pH 6.8), 10\% glycerol, $5 \% 2$-mercaptoethanol, $2 \%$ SDS, $0.01 \%$ bromophenol blue and 5 mM EDTA. SDS-PAGE was performed on cell lysate samples and separated proteins were electrotransferred to membrane filters (Immobilon-P; EMD Millipore). Subsequent to blocking with TBS-T (10 mM Tris-HCl, pH 7.6, $150 \mathrm{mM}$ sodium chloride $0.1 \%$ Tween-20) containing $5 \%$ bovine serum albumin (BSA), filters were incubated with primary antibodies in TBS-T containing 2\% BSA overnight. Filters were washed with TBS-T and incubated for $1 \mathrm{~h}$ in horseradish peroxidase-conjugated anti-mouse or anti-rabbit IgG (NA931 and NA934, respectively; GE Healthcare Life Sciences, Chalfont, UK) diluted 1:10,000 in TBS-T containing $2 \%$ BSA. Subsequent to several washes with TBS-T, immunoreactivity was detected using an enhanced chemiluminescence system (GE Healthcare Life Sciences) according to the manufacturer's protocol.

Statistical analysis. All quantitative data are presented as means \pm standard deviation estimated from $\geq 3$ replicates per experiment. Student's t-test and Fisher's exact probability test were used for statistical analyses. All statistical analyses were performed using the $\mathrm{R}$ statistical software package, version 2.6.2 ( $\mathrm{R}$ foundation for Statistical Computing, Vienna, Austria). $\mathrm{P}<0.05$ was considered to indicate a statistically significant result.

\section{Results}

Periostin suppresses the in vitro invasiveness of the human bladder cancer UMUC-3 cell line. To investigate the ability of periostin to suppress the in vivo malignant phenotypes of bladder cancer cells in a mouse orthotopic model, the human bladder cancer UMUC-3 cell line was used. An amphotropic retrovirus containing the blasticidin-resistant gene and the periostin gene tagged with Flag at its C-terminus (pCXbsr/Per) and the control vector virus (pCXbsr) were introduced into UMUC-3 cells. RT-PCR and immunoblot analyses demonstrated that UMUC-3 cells infected with pCXbsr/Per expressed exogenous periostin $\mathrm{mRNA}$ and protein (Fig. 1A and B). The growth rate of UMUC-3 cells expressing exogenous periostin was similar to that of control cells infected with the vector virus (Fig. 1C). The tumorigenicity of the two cell lines was assayed by subcutaneous injection into nude mice. As shown in Fig. 1D, three weeks following inoculation, the mean tumor weight from three mice inoculated with periostin-expressing UMUC-3 cells $(0.56 \pm 0.31 \mathrm{~g})$ were almost similar to that from three mice inoculated with vector control UMUC-3 cells $(0.46 \pm 0.26 \mathrm{~g} ; \mathrm{P}=0.691)$. However, the in vitro cell invasiveness of UMUC-3 cells expressing exogenous periostin was markedly lower compared with control cells infected with the vector virus (Fig. 1E). These results indicate that periostin is able to suppress the in vitro cell invasiveness of UMUC-3 bladder cancer cells without affecting cellular proliferation and subcutaneous tumor growth in nude mice.

Periostin suppresses in vivo invasiveness of UMUC-3 cells in a mouse orthotopic model of bladder cancer. To assess the ability of periostin to suppress the in vivo invasiveness and tumorigenicity of UMUC-3 cells, vector control and periostin-expressing UMUC-3 cells were instilled into the bladders of seven (group A) and nine (group B) nude mice, respectively. As shown in Table I, no differences in body weight were observed between the two groups $(\mathrm{P}=0.335) 4$ weeks subsequent to the instillation of bladder cancer cells. The mean weight of bladders instilled with periostin-expressing UMUC-3 cells (group B, 39.4 $\pm 11.0 \mathrm{mg}$ ) was significantly lower than bladders instilled with vector control cells (group A, $61.7 \pm 20.0 \mathrm{mg}, \mathrm{P}=0.013)$. Tumor formation was detected in six of seven $(85.7 \%)$ and five of nine $(55.6 \%)$ bladders in vector control UMUC-3 cells (group A) and periostin-expressing UMUC-3 cells (group B), respectively. A total of five $(83.3 \%$ ) of 6 UMUC-3 bladder tumors containing the vector virus exhibited histopathological evidence of muscle invasion (Fig. 2A), while none of the five periostin-expressing UMUC-3 bladder tumors revealed muscle invasion ( $\mathrm{P}=0.031$, Fig. $2 \mathrm{~B}$ ). Thick edematous lesions were observed in the submucosa of all periostin-expressing UMUC-3 bladder tumors (Fig. 2B-D). Tumors were separated from muscular layers by these edematous lesions in periostin-expressing UMUC-3 bladder tumors. 
Table I. Results of the mouse orthotopic model of bladder cancer using UMUC-3 cells.

\begin{tabular}{|c|c|c|c|}
\hline Characteristics & Control & Periostin & P-value \\
\hline $\mathrm{n}$ & 7 & 9 & \\
\hline Body weight, $\mathrm{g}^{\mathrm{a}}$ & $23.8 \pm 1.6$ & $24.4 \pm 1.0$ & 0.335 \\
\hline Bladder weight, mga & $61.7 \pm 20.0$ & $39.4 \pm 11.0$ & 0.013 \\
\hline Tumor formation ${ }^{\mathrm{b}}$ & $6 / 7(85.7 \%)$ & $5 / 9(55.6 \%)$ & 0.455 \\
\hline Bladder weight of tumor formed mouse, $\mathrm{mg}^{\mathrm{a}}$ & $66.7 \pm 16.3$ & $46.6 \pm 9.7$ & 0.039 \\
\hline Muscle invasion of bladder tumor ${ }^{b}$ & $5 / 6(83.3 \%)$ & $0 / 5(0 \%)$ & 0.031 \\
\hline Mitotic activity index ${ }^{a}$ & $12.0 \pm 3.0$ & $11.9 \pm 3.5$ & 0.974 \\
\hline
\end{tabular}

${ }^{\mathrm{a}}$ Mean \pm standard deviation ${ }^{\mathrm{b}} \mathrm{n}(\%)$.

A

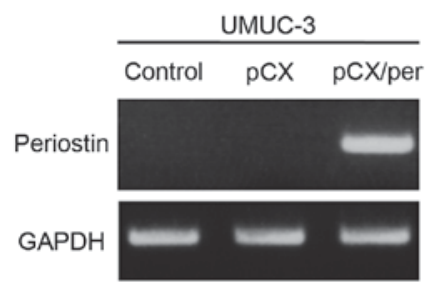

C

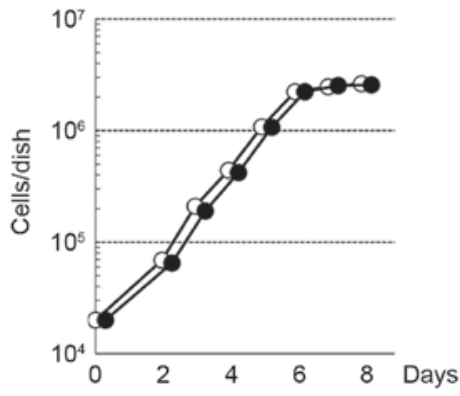

B

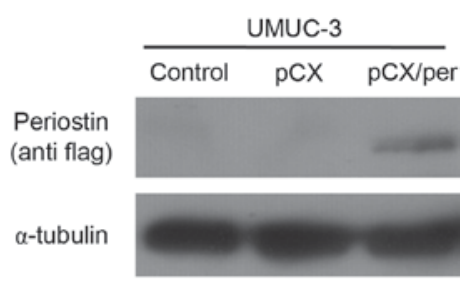

D

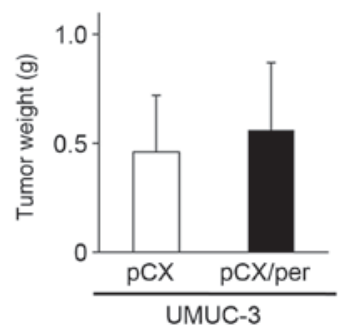

E

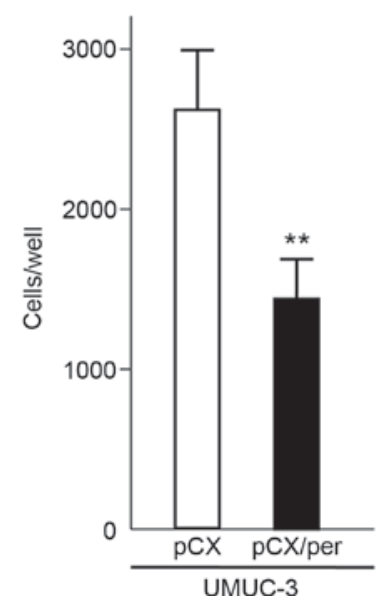

Figure 1. Effect of ectopic expression of periostin on cell invasiveness, cellular proliferation and subcutaneous tumor growth in UMUC-3 cells. (A) RT-PCR analysis of periostin in UMUC-3 cells infected with pCX or pCX/Per. GAPDH expression was used as an internal control. (B) Immunoblot analysis of exogenous periostin in UMUC-3 cells infected with pCX or pCX/Per. Exogenous expression of periostin was detected by anti-Flag antibody. $\alpha$-tubulin was used as an internal control. (C) Growth curves of UMUC-3 cells infected with vector virus or periostin-expressing virus. Experiments were performed in triplicate. SDs were too small to show using error bars. (D) Subcutaneous tumor growth of UMUC-3 cells infected with vector virus or periostin-expressing virus. $\mathrm{Bar} \pm \mathrm{SD}$ of three mice for each cell line. $\mathrm{P}=0.691$. (E) Suppression of cell invasiveness of UMUC-3 cells by periostin expression. Number of cells invading through the Matrigel is shown. Each sample was assayed in triplicate. Bar \pm SD of triplicate chambers for each experiment. ${ }^{* *} \mathrm{P}=0.006$ vs. pCX. pCX, control virus; $\mathrm{pCX} / \mathrm{Per}$, periostin-expressing virus; white circle, vector virus; black circle, periostin-expressing virus; SD, standard deviation; white bar, vector virus; black bar, periostin-expressing virus. 

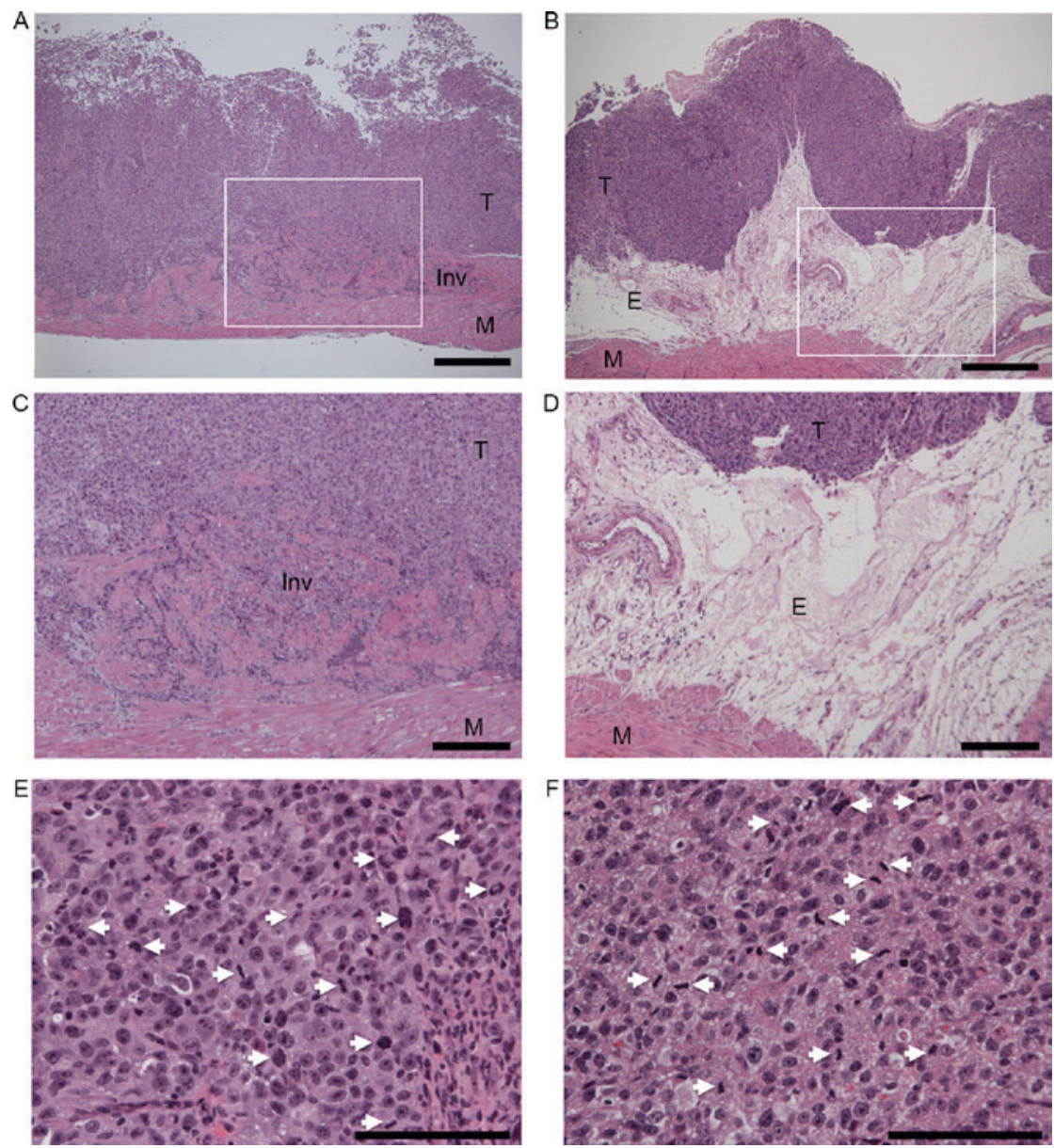

Figure 2. Histopathological examinations of (A) vector control and (B) periostin-expressing UMUC-3 bladder tumors in a mouse orthotopic model of bladder cancer. Hematoxylin and eosin (HE) stain, magnification x40. Scale bars represent $500 \mu \mathrm{m}$. (C and D) are higher magnification of (A and B), respectively. White squares in (A and B) indicate the area of (C and D), respectively. (C) Invasive growth of vector control UMUC-3 bladder tumor. (D) Edematous lesions of periostin-expressing UMUC-3 bladder tumor (magnification, x100). Scale bars represent $200 \mu \mathrm{m}$. (E) Mitotic figures in vector control and (F) periostin-expressing UMUC-3 bladder tumors (magnification, $\mathrm{x} 400$ ). Scale bars represent $100 \mu \mathrm{m}$. The white arrows indicate mitotic cells. Mitotic counts were performed using a standard laboratory microscope (magnification, x40). Mitotic activity index (mitotic figures/3 hpfs) of mouse orthotopic bladder tumors was determined using the number of mitotic figures in three randomly-selected hpfs. T, tumor; M, muscular layer; E, edematous lesion; Inv, Invasive growth; hpfs, high power fields.

Even in the bladder tumor-formed mice, the mean weight of bladders instilled with periostin-expressing UMUC-3 cells $(46.6 \pm 9.7 \mathrm{mg}$ ) was significantly lower compared with bladders instilled with vector control cells $(66.7 \pm 16.3 \mathrm{mg}, \mathrm{P}=0.039)$. As no difference in body weights was observed between the two groups, the differences in bladder weight were likely a result of bladder tumor size. No differences in the mitotic activity index, an indicator of proliferation, were observed between control vector and periostin-expressing UMUC-3 bladder tumor tissues (Table I, Fig. 2E and F). These results indicate that periostin is able to suppress the in vivo invasiveness and tumor growth of UMUC-3 bladder tumors without affecting proliferative activity in a mouse orthotopic model of bladder cancer.

Effect of periostin on Akt and mTOR signaling in UMUC-3 cells. To investigate the underlying mechanism of periostin to suppress invasion, the expression of E-cadherin was examined. As shown in Fig. 3A, E-cadherin expression was not detected in periostin-expressing and vector control UMUC-3 cells. No differences in the expression of Snail or Twist, known to negatively regulate E-cadherin transcription, were observed between vector control and periostin-expressing UMUC-3 cells. The association between the expression of periostin and signaling of Akt-mTOR in bladder cancer was also determined. As shown in Fig. 3B, exogenous expression of periostin decreased Akt phosphorylation in UMUC-3 cells. Measurement of phosphorylation of S6 ribosomal protein, a downstream protein of mTOR kinase, was used to determine mTOR activity. S6 phosphorylation was also found to be decreased in periostin-expressing UMUC-3 cells compared with control cells (Fig. 3B). Phosphorylation of PDK1, an upstream kinase of Akt, was also suppressed in periostin-expressing UMUC-3 cells (Fig. 3B). However, phosphorylation of PI3 K, another upstream kinase of Akt, was not detected in periostin-expressing and control UMUC-3 cells, although PI3 K protein was faintly expressed in the two types of cell (Fig. 3B). Together, these results indicate that periostin is able to suppress the activity of the PDK1/Akt/mTOR pathway in UMUC-3 cells.

Treatment with $r$-periostin suppresses cell invasiveness and PDK1/Akt/mTOR signaling in UMUC-3 cells. The present 
A

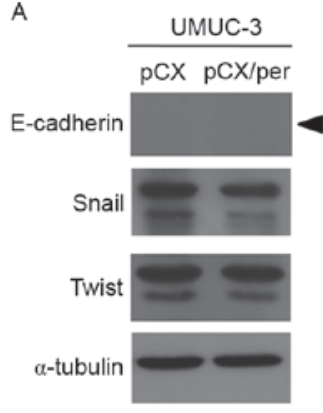

D

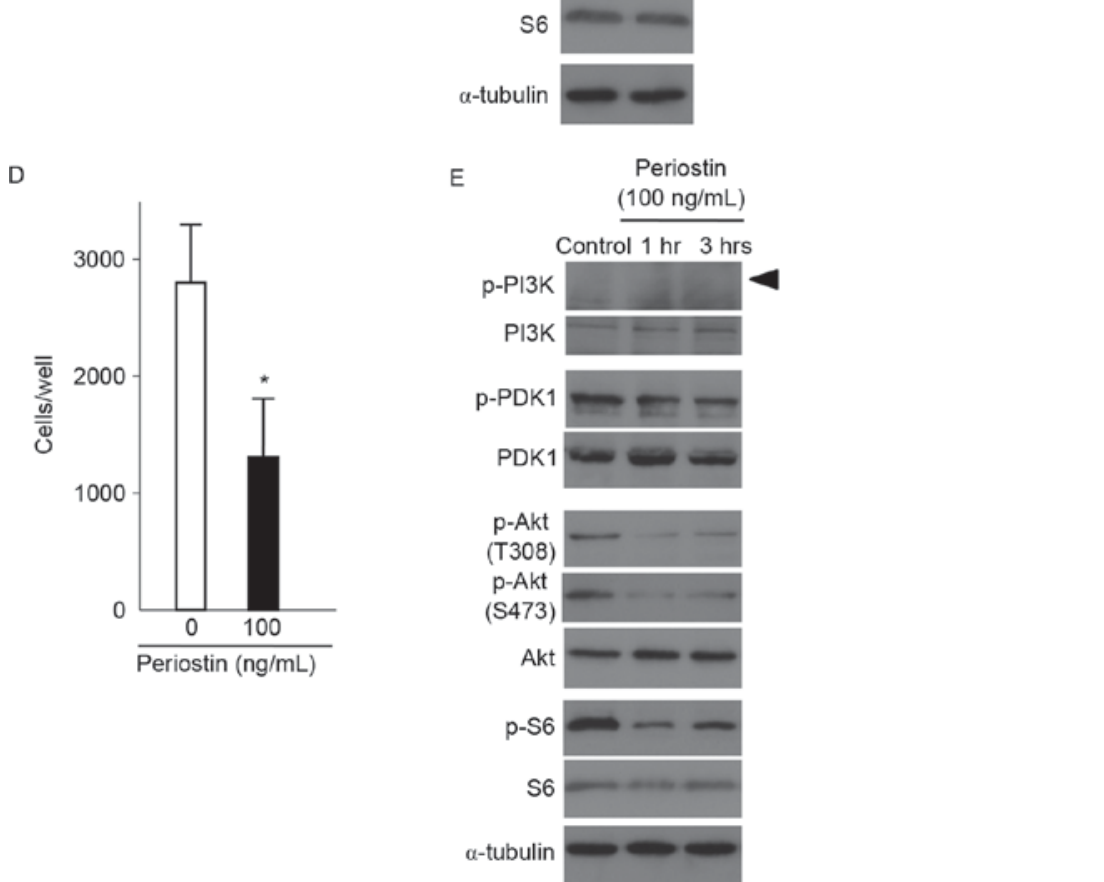

B

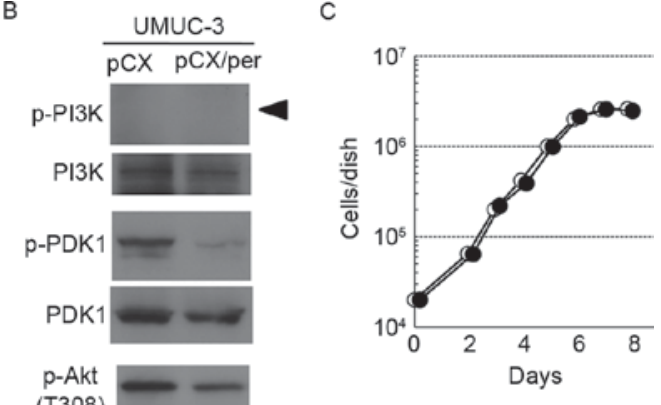

Figure 3. Effects of the expression of periostin on PDK1/Akt/mTOR signaling and expression of proteins regulating epithelial-mesenchymal transition in UMUC-3 cells. (A) Immunoblot analyses of the EMT-associated proteins E-cadherin, Snail and Twist in vector control and periostin-expressing UMUC-3 cells. $\alpha$-tubulin expression was used as an internal control. The arrowhead indicates the E-cadherin band. (B) Immunoblot analyses of total and phosphorylated forms of PDK1, Akt and S6, a downstream protein of mTOR, in vector control and periostin-expressing UMUC-3 cells. $\alpha$-tubulin expression was used as an internal control. The arrowhead indicates the p-PI3K band. (C) Growth curves of UMUC-3 cells treated with 100 ng/ml periostin and vector control cells. Experiments were performed in triplicate. SD was too small to show as error bars. White and black circles indicate the control and periostin treatment (100 ng/ml), respectively. (D) Suppression of cell invasiveness of UMUC-3 cells following treatment with $100 \mathrm{ng} / \mathrm{ml}$ periostin for 6 h. Number of cells invading through the Matrigel is shown. Bar, \pm SD of triplicate chambers for each experiment. * $\mathrm{P}=0.046 \mathrm{vs}$. control cells. (E) Immunoblot analyses of total and phosphorylated forms of PDK1, Akt and S6 in control UMUC-3 cells and cells treated with $100 \mathrm{ng} / \mathrm{ml}$ periostin for 1 and $3 \mathrm{~h}$. $\alpha$-tubulin expression was used as an internal control. The arrowhead indicated the p-PI3K band. PDK1, phosphoinositide-dependent kinase-1; Akt, protein kinase B; mTOR, mammalian target of rapamycin; SD, standard deviation.

study assessed the effect of treatment with r-periostin on cell invasiveness and PDK1/Akt/mTOR signaling. The UMUC-3 cells were treated with $100 \mathrm{ng} / \mathrm{ml}$ of r-periostin. As revealed in Fig. 3C and D, r-periostin treatments suppressed in vitro cell invasiveness without affecting cellular proliferation. As shown in Fig. 3E, phosphorylation of PDK1, Akt and S6 was also suppressed following treatment with r-periostin for 1 and $3 \mathrm{~h}$. These results are consistent with the results of experiments using periostin-expressing UMUC-3 cells (Figs. 1, 3A and B).

Effects of PDK1, Akt, and mTOR inhibition on in vitro cell invasiveness of UMUC-3 cells. To assess the contribution of
PDK1/Akt/mTOR signaling to the suppression of cell invasiveness by periostin, the present study evaluated the effects of inhibitors of PDK1 $(0.1$ and $1 \mu \mathrm{M})$, Akt $(10$ and $25 \mu \mathrm{M})$ and mTOR ( 5 and $20 \mathrm{nM}$ ) on the invasiveness of UMUC-3 cells. Immunoblot analysis demonstrated that respective inhibitors effectively suppressed the phosphorylation of PDK1, Akt and S6 without affecting PDK1, Akt and S6 protein levels (Fig. 4A-C). Akt inhibitors also suppressed the phosphorylation of S6. Following incubation with inhibitors for $6 \mathrm{~h}$, cells were inoculated into Matrigel chambers without the inhibitors. As shown in Fig. 4D-F, treatment with each of the inhibitors significantly suppressed the in vitro cell invasiveness of 

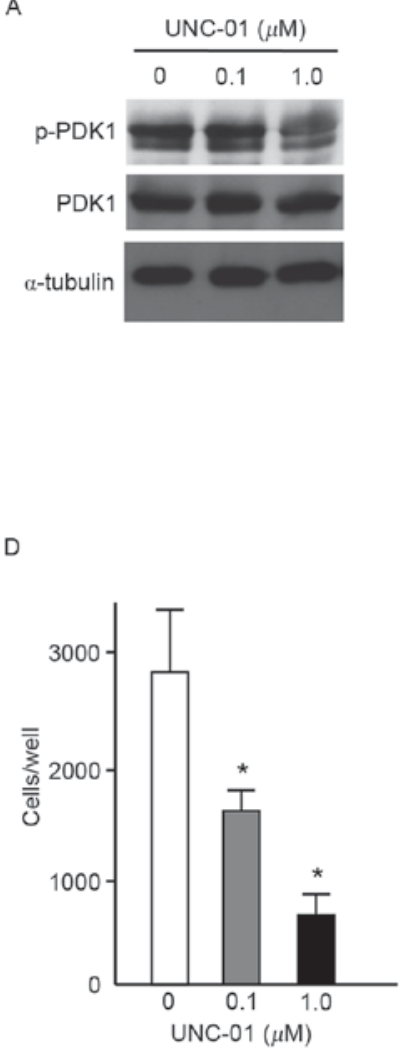

G

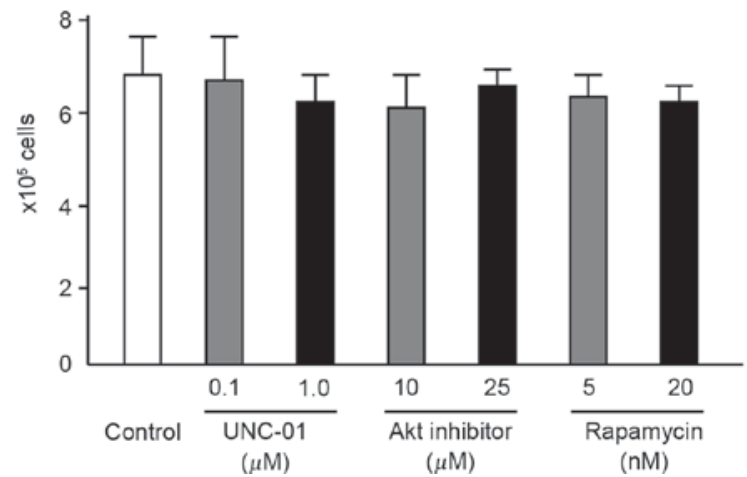

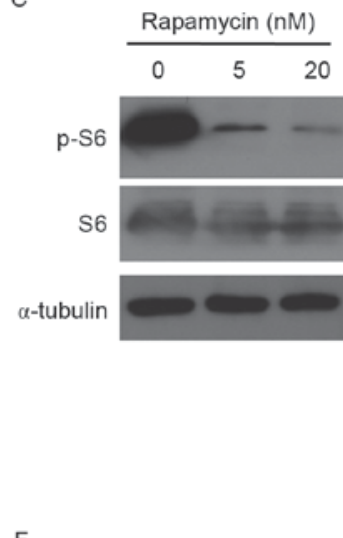
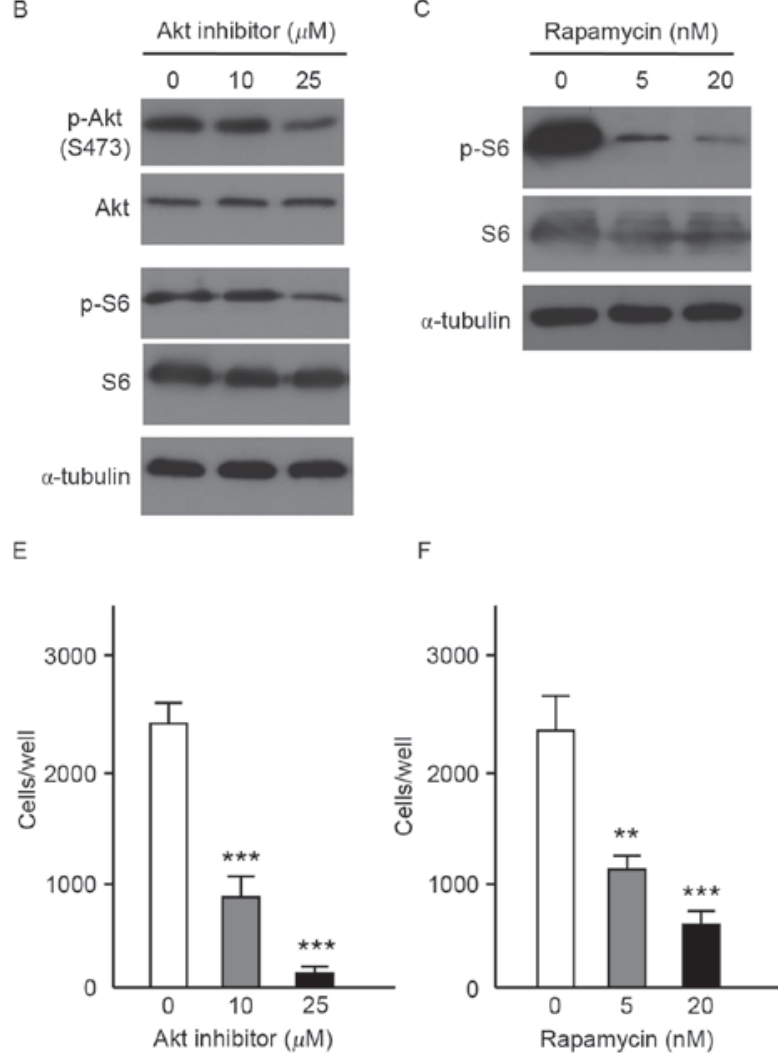

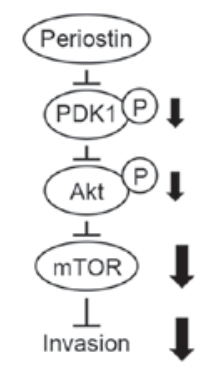

Figure 4. Effects of PDK1, Akt and mTOR inhibitors on the in vitro cell invasiveness of UMUC-3 cells. (A) Immunoblot analysis of UMUC-3 cells treated with (A) the PDK1 inhibitor, UNC-01 (0.1 and $1 \mu \mathrm{M})$, (B) Akt inhibitor (10 and $25 \mu \mathrm{M})$ and (C) the mTOR inhibitor, rapamycin (5 and 20 nM). Expression of $\alpha$-tubulin was used as an internal control. Effect of (D) PDK1 inhibitor, (E) Akt inhibitor and (F) mTOR inhibitor on the cell invasiveness of UMUC-3 cells. Number of cells invading through the Matrigel is shown. Each sample was assayed in triplicate. Bars, \pm SD of triplicate chambers for each experiment. ${ }^{*} \mathrm{P}<0.05,{ }^{* *} \mathrm{P}=0.003,{ }^{* * *} \mathrm{P}<0.001$ vs. untreated control cells. (G) Cell proliferation of untreated control UMUC-3 cells and UMUC-3 cells treated with PDK1, Akt and mTOR inhibitors. Cells grown in triplicate plates were trypsinized and counted. Bars, \pm SD of triplicate culture dishes for each experiment. (H) Schematic illustration of the signaling pathway responsible for the suppressive effect of periostin on the invasiveness of UMUC-3 bladder cancer cells. PDK1, phosphoinositide-dependent kinase-1; Akt, protein kinase B; mTOR, mammalian target of rapamycin; SD, standard deviation.

UMUC-3 cells. No effect on cellular proliferation of UMUC-3 cells was observed with any of the inhibitors (Fig. 4G). These results indicate that $\mathrm{PDK} 1 / \mathrm{Akt} / \mathrm{mTOR}$ signaling is required for the suppression of cell invasiveness by periostin in UMUC-3 cells (Fig. 4H).

\section{Discussion}

The present study demonstrated that periostin is able to suppress the in vivo invasiveness of bladder cancer cells in a mouse orthotopic model system. Although the difference in in vitro and in vivo proliferation was not observed between the control vector and periostin-expressing UMUC-3 cells, periostin-expressing UMUC-3 bladder tumors were smaller compared with the control UMUC-3 bladder tumors. The suppression of tumorigenicity by periostin may be due to decreased numbers of cancer cells implanting into the bladder surface, as periostin-expressing UMUC-3 bladder tumors exhibited reduced invasive ability. Notably, thick edematous lesions were observed in the submucosa of periostin-expressing UMUC-3 bladder tumors. The development of thick edematous lesions in response to periostin may contribute to the 
suppression of tumor growth and invasion. However, the mechanism underlying the formation of edematous lesions in the submucosa of periostin-expressing UMUC-3 bladder tumors has yet to be elucidated. Edematous lesions were not detected in periostin-expressing UMUC-3 subcutaneous tumors in nude mice, and no difference in tumorigenicity was observed between the control vector and periostin-expressing UMUC-3 subcutaneous tumors in nude mice. Opposing effects of periostin on differing cancer types suggest tissue-specific functions of periostin (9). Organ-specific fibroblasts may contribute to the suppressive effects of periostin on in vivo tumor invasiveness.

Furthermore, the present study revealed that the PDK1/Akt/mTOR signaling pathway is required for the suppressive effects of periostin on the invasiveness of UMUC-3 cells. We previously identified the suppression of in vitro cell invasiveness by periostin in the human bladder cancer cell lines, SBT31A and T24. In these cells, periostin upregulated the expression of E-cadherin via the suppression of Akt phosphorylation and Twist (9). However, ectopic expression of periostin in UMUC-3 bladder cancer cells did not induce E-cadherin expression. Suppression of PI3K activity via a negative feedback mechanism may be responsible for the differing responses between T24 and UMUC-3 cells as a result of the homozygous null deletion of PTEN in UMUC-3 cells (14). Alterations of PTEN, encompassing homozygous gene deletion, loss of heterozygosity, and gene mutation, have been identified in $\sim 30 \%$ of patients with bladder cancer (15). However, the effect of PTEN inactivation on downstream signal cascades, such as mTOR, in bladder cancer has not yet been fully evaluated. UMUC-3 cells exhibit utility in elucidating the molecular mechanisms underlying carcinogenesis in bladder cancer cells deficient in PTEN. The PI3K/Akt/mTOR signaling pathway has recently been identified as a candidate pathway in bladder cancer progression by clinicopathological studies $(14,16)$. mTOR-containing multiprotein complex 1 (mTORC1) regulates cell growth, proliferation and survival, whereas mTORC2 controls cytoskeletal remodeling and cancer cell adhesion and migration $(17,18)$. Previous studies have demonstrated that rapamycin-sensitive mTORC1 serves a critical role in the regulation of cell motility and invasion (19-21). In the present study, rapamycin clearly suppressed the invasiveness of UMUC-3 cells, indicating that mTORC1 is required for the suppressive effects of periostin on the cell invasiveness of bladder cancer. The PDK1/Akt/mTOR signaling pathway may constitute a targetable signaling pathway for therapies against bladder cancer invasion.

In clinical cases, non-muscle invasive bladder cancer is predominantly treated by transurethral resection of bladder tumor (TUR-Bt). However, the intravesical recurrence rate of bladder cancer following TUR-Bt has been revealed to be $50-70 \%(22,23)$. The majority of the recurrence of bladder cancer is recognized as the result of intraluminal seeding. Surgical injury of TUR-Bt disseminates a large number of cancer cells, and forms the mucosal defect, resulting in the implantation of cancer cells in the bladder surface $(24,25)$. Therefore, the prevention of early seeding following TUR-Bt is crucial. Indeed, a single intravesical instillation of a chemotherapeutic agent immediately following TUR-Bt significantly decreased the risk of recurrence in patients with bladder cancer $(26,27)$. In the present study, cancer cells were instilled into bladders following trypsin treatment of the bladder surface in a mouse orthotopic model of bladder cancer. This system resembles the implantation of cancer cells subsequent to TUR-Bt. The invasiveness of cancer cells is closely associated with tumor cell seeding. In the present study, periostin was revealed to suppress the invasiveness and tumorigenicity of cancer cells in a mouse orthotopic model of bladder cancer. This suppression may result from the decreased number of the implanted cancer cells on bladder surface by the function of periostin. Treatment with r-periostin $(100 \mathrm{ng} / \mathrm{ml})$ was able to suppress the in vitro invasiveness of bladder cancer cells via modulation of PDK1/Akt/mTOR signaling. Periostin may exhibit utility as a potent chemotherapeutic agent for use in intravesical treatment following TUR-Bt by suppressing bladder cancer invasiveness.

\section{Acknowledgements}

The authors would like to thank Ms. Akiyo Ushio (Division of Microbiology and Infectious Diseases, Shiga University of Medical Science) for her technical assistance. The present study was supported by a Grant-in Aid for Scientific Research (C) (grant nos. 25462476 and 24590480) from the Ministry of Education, Science, Sports, and Culture of Japan.

\section{References}

1. Hwang EY, Jeong MS, Park EK, Kim JH and Jang SB: Structural characterization and interaction of periostin and bone morphogenetic protein for regulation of collagen cross-linking. Biochem Biophys Res Commun 449: 425-431, 2014.

2. Norris RA, Borg TK, Butcher JT, Baudino TA, Banerjee I and Markwald RR: Neonatal and adult cardiovascular pathophysiological remodeling and repair: Developmental role of periostin. Ann N Y Acad Sci 1123: 30-40, 2008.

3. Horiuchi K, Amizuka N, Takeshita S, Takamatsu H, Katsuura M, Ozawa H, Toyama Y, Bonewald LF and Kudo A: Identification and characterization of a novel protein, periostin, with restricted expression to periostium and periodontal ligament and increased expression by transforming growth factor beta. J Bone Miner Res 14: 1239-1249, 1999.

4. Katsuragi N, Morishita R, Nakamura N, Ochiai T, Taniyama Y, Hasegawa Y, Kawashima K, Kaneda Y, Ogihara T and Sugimura K: Periostin as a novel factor responsible for ventricular dilation. Circulation 110: 1806-1813, 2004.

5. Wang D, Oparil S, Feng JA, Li P, Perry G, Chen LB, Dai M, John SW and Chen TF: Effects of pressure overload on extracellular matrix expression in the heart of the atrial natriuretic peptide-null mouse. Hypertension 42: 88-95, 2003.

6. Wilde J, Yokozeki M, Terai K, Kudo A and Moriyama K: The divergent expression of periostin mRNA in the periodontal ligament during experimental tooth movement. Cell Tissue Res 312: 345-351, 2003.

7. Ruan K, Bao S and Ouyang G: The multifaceted role of periostin in tumorigenesis. Cell Mol Life Sci 66: 2219-2230, 2009.

8. Kim CJ, Yoshioka N, Tambe Y, Kushima R, Okada Y and Inoue $\mathrm{H}$ : Periostin is down-regulated in high grade human bladder cancers and suppresses in vitro cell invasiveness and in vivo metastasis of cancer cells. Int J Cancer 117: 51-58, 2005.

9. Kim CJ, Sakamoto K, Tambe Y and Inoue H: Opposite regulation of epithelial-to-mesenchymal transition and cell invasiveness by periostin between prostate and bladder cancer cells. Int $\mathrm{J}$ Oncol 38: 1759-1766, 2011.

10. Watanabe T, Shinohara N, Sazawa A, Harabayashi T, Ogiso Y, Koyanagi T, Takiguchi M, Hashimoto A, Kuzumaki N, Yamashita M, et al: An improved intravesical model using human bladder cancer cell lines to optimize gene and other therapies. Cancer Gene Ther 7: 1575-1580, 2000. 
11. Tanaka M, Gee JR, De La Cerda J, Rosser CJ, Zhou JH, Benedict WF and Grossman HB: Noninvasive detection of bladder cancer in an orthotopic murine model with green fluorescence protein cytology. J Urol 170: 975-978, 2003.

12. Kim CJ, Isono T, Tambe Y, Chano T, Okabe H, Okada $\mathrm{Y}$ and Inoue $\mathrm{H}$ : Role of alternative splicing of periostin in human bladder carcinogenesis. Int J Oncol 32: 161-169, 2008.

13. Naviaux RK, Costanzi E, Haas M and Verma IM: The pCL vector system: Rapid production of helper-free, high-titer, recombinant retroviruses. J Virol 70: 5701-5705, 1996.

14. Hansel DE, Platt E, Orloff M, Harwalker J, Sethu S, Hicks JL, De Marzo A, Steinle RE, His ED, Theodorescu D, et al: Mammalian target of rapamycin (mTOR) regulates cellular proliferation and tumor growth in urothelial carcinoma. Am J Pathol 176: 3062-3072, 2010.

15. Gildea JJ, Herlevsen M, Harding MA, Gulding KM, Moskaluk CA, Frierson HF and Theodorescu D: PTEN can inhibit in vitro organotypic and in vivo orthotopic invasion of human bladder cancer cells even in the absence of its lipid phosphatase activity. Oncogene 23: 6788-6797, 2004.

16. Sun $\mathrm{CH}$, Chang $\mathrm{YH}$ and Pan CC: Activation of the $\mathrm{PI} 3 \mathrm{~K} / \mathrm{Akt} / \mathrm{mTOR}$ pathway correlates with tumour progression and reduced survival in patients with urothelial carcinoma of the urinary bladder. Histopathology 58: 1054-1063, 2011.

17. Wan X, Mendoza A, Khanna C and Helman LJ: Rapamycin inhibits ezrin-mediated metastatic behavior in a murine model of osteosarcoma. Cancer Res 65: 2406-2411, 2005.

18. Busch S, Renaud SJ, Schleussner E, Graham CH and Markert UR mTOR mediates human trophoblast invasion through regulation of matrix-remodeling enzymes and is associated with serine phosphorylation of STAT3. Exp Cell Res 315: 1724-1733, 2009.

19. Berven LA, Willard FS and Crouch MF: Role of the p70(S6K) pathway in regulating the actin cytoskeleton and cell migration. Exp Cell Res 296: 183-195, 2004.
20. Liu L, Li F, Cardelli JA, Martin KA, Blenis J and Huang S: Rapamycin inhibits cell motility by suppression of mTOR-mediated S6K1 and 4E-BP1 pathways. Oncogene 25: 7029-7040, 2006.

21. Liu L, Chen L, Chung J and Huang S: Rapamycin inhibits F-actin reorganization and phosphorylation of focal adhesion proteins. Oncogene 27: 4998-5010, 2008.

22. Lutzeyer W, Rübben H and Dahm H: Prognostic parameters in superficial bladder cancer: An analysis of 315 cases. J Urol 127: 250-252, 1982.

23. Herr HW, Laudone VP and Whitmore WF Jr: An overview of intravesical therapy for superficial bladder tumors. J Urol 138: 1363-1368, 1987.

24. Soloway MS and Masters S: Urothelial susceptibility to tumor cell implantation: Influence of cauterization. Cancer 46: 1158-1163, 1980

25. See WA, Miller JS and Williams RD: Pathophysiology of transitional tumor cell adherence to sites of urothelial injury in rats: Mechanisms mediating intravesical recurrence due to implantation. Cancer Res 49: 5414-5418, 1989.

26. Sylvester RJ, Oosterlinck W and van der Meijden AP: A single immediate postoperative instillation of chemotherapy decreases the risk of recurrence in patients with stage Ta T1 bladder cancer: A meta-analysis of published results of randomized clinical trials. J Urol 171: 2186-2190, 2004.

27. Okamura K, Ono Y, Kinukawa T, Matsuura O, Yamada S, Ando T, Fukatsu T, Ohno Y, Ohshima S; Nagoya University Urological Oncology Group: Randomized study of a single early instillation of (2"R)-4'-O-tetrahydropyranyl-doxorubicin for a single superficial bladder carcinoma. Cancer 94: 2363-2368, 2002. 